\title{
A Toolbox Perspective on Chinese-Self
}

\author{
Tong Yumeng ${ }^{1 *}$ \\ ${ }^{1}$ Department of English, University of Macau, Avenida da Universidade, Taipa, Macau 999078, China \\ *Corresponding author. Email: ab61768@umac.edu.mo
}

\begin{abstract}
Chinese self-understanding is one of the major themes with the rising of Neo-Confucianism. After Zhu Xi and Wang Yangming, many generations of Confucian scholars have proposed different models concerning the topic. They challenge, develop from, contradict each other, and sometimes with minor consensus; their approaches occasionally intend to solve the issue metaphysically while sacrifice their practicality. In this paper, it will firstly identify the problems of the model proposed by the currently most famous role-relational school. This model, on the one hand, due to the unpopularity of Confucianism in Post-modern China, suffers from a practical predicament; on the other hand, since it heavily relies on moral exemplary and intuition, the method of being a role-bearing person is vague. The two types of role-dilemma are examples of the symptoms caused by the above obscurity. To diagnose these issues, it will adopt motivational theories in psychology to point out their pathological dynamics. The Self-Determination theory and the attributional theory might delineate two illustrations, both cognitively and emotively, on how the Chinese-self gets stuck in cold principles thus loses its creativity. Therefore, in the Third part of this paper, it will propose a toolbox approach to combine several recognized models on Chinese-self since the revival of neo-Confucianism: the rational concept of $L i$ in Zhu Xi's theory; the sentimental Xin in Wang Yangming's doctrine; the focus-field relation from Roger Ames' terminology and the genealogical self in Wang Qingjie's reconstruction. To unify them harmoniously, it will review Fung Yu-lan's two types of mythology, the positive method and the negative method, on Chinese philosophy, and indicates its metaphysical limits in his four spheres. To ameliorate his idea, this paper suggests a dialectic solely within the ethical or interpersonal sphere through his two methods, by which two rules on the formation of the Chinese-self are the natural results: one is on a cognitive aspect; another is on an emotive aspect. The guiding lines of them are from the previous concept of $L i$ and $X i n$; the dynamics of them is through the focus-field interaction; and the boundary of them is demonstrated by the analogy of genealogy on Chinese-characters.
\end{abstract}

Keywords: Chinese-self, Confucianism, Neo-Confucianism, Role-relations, Self-Determination Theory, Attributional Theory, Fung Yu-lan, Recognition, Resonance.

\section{PROBLEMS IDENTIFICATION}

As Professor Wang [1] points out in his article, before raising his thesis on the "genealogical self", the relational-self based on social roles, when abandoning the previous obsessions on metaphysical universality, seems to be closer to the true Chinese self-perception. Professor King [2] is one of the defenders of this relational-self. As what he illustrates, this relational-self contains the notion of "self-centered voluntarism" but this voluntarism does not mean the self is free. In Chinese ethical structures, as he argues, centralized social relations are in two categories: "preordained given" and "voluntarily constructed". The preordained relations perhaps originate from the Confucian five cardinal relations. It grids Chinese social consciousness and becomes an unconditional norm. However, the New Culture Movement complicates its authority. With its revolutionary radicality, it, on the one hand, yearns to drastically negate this Confucian foundation; nevertheless, on the other hand, the culture inertia remains. It seems that this Five-Cardinal relational perception is so internalized that the conscious resistance pales at the unconsciousness reminiscence. Consequently, as King laments at the end of his article: the five-cardinal based relations "are not universal and comprehensive in nature". Worse still, they tend to unmask apathetic behaviors toward strangers. If considering the tension between five cardinal relations and the voluntarism in relational-self, one might notice that: if King's analysis is true, the voluntarism nature in Confucian role-relations might compensate the limits in five cardinal relations. For 
example, an individual might "creatively" extend his/her Five Cardinal relations by regarding strangers as quasifriends as what Confucius famously says "all within the four seas will be his brothers" [3].

\subsection{The Practical Problem}

It perhaps betrays that the five cardinal relations decayed as soulless principles and loses popularity. As what $\mathrm{Ci}$ incisively remarked recently, in one aspect, this guan xi ben wei (關係本位) society, as the derivative of five cardinal relations, degrades into "the corruption of the entire people" [4]. From this perspective, how to revitalize this outdated principle in the postmodern Chinese-self is perhaps the most urgent practical problem in the project of self-formation. This essay will address it later.

\subsection{The Methodological Problem}

Another role-relational school seems to try to abandon this five-cardinal ground.

\subsubsection{Roger Ames'Relational Model}

The exemplary representative perhaps is Ames. Inspired by Tang Junyi's [5] interpretation of Book of Changes, he postulates that the Chinese role ethics offer "guidelines" rather than "abstract principles". The nature of Chinese-self, when represented in its relations, as far as what I understand of him, is "organistic amorphous".

In the first place, "the part may be a functionally interrelated element helping to constitute an organism" [6]; but companying with it, the realization of this aspiration is "in an ever-changing context over the conceptualization and articulation of these pursuits". Therefore, in grasping the notion of human xing (性), we need to know it "is formed continuously as the opportunity presents itself" [7]. Following the above premises, Ames suggests a holographic interpretation of this role-self construct. The essence of it, firstly, is the inseparable co-existence between its two parts: the field and the focuses. Both the field and the focus are equally crucial. Every behavior in each role triggers a focus and the energy of it radiates a field which in resonance with other focal-fields (or the whole field itself).

Based on that, the second feature is that this coherent structure is correlative. Ames and Hall reject Hansen's "mereological model" because it dedicates to the "recognition or establishment of boundaries" [8] under the notion of zheng ming. However, in their point of view, ming, as the "definition" of each role ethically, might not be that analytically clear-cut nor it should be. The guidelines for each person to enact their roles are closer to an aesthetic play rather than rigid execution. In such a way, Ames qualifies his two-categorical social relations by emphasizing the one under voluntary interaction over the inflexible one influenced by the five cardinal relations.

\subsubsection{Henry Rosemont's Development}

Based on this decentralized associative view, Rosemont's model of "the onion self" might be an interpretation to the abstract holographic view. He regards Chinese as "role-bearing persons" by saying that:

“Moreover, we do not 'play those roles'... but rather live our roles ... Being thus the aggregate sum of the roles I live, it must follow that as I grow older my role will change, and consequently I become quietly literally a different person." [9].

Rosemont's book appeals to western readers. Thus, to make this west-east comparison, he develops the contrast between Western peach-self and Chinese onion-self. The central difference between the two is on whether there is a centralized core in self-conceptualization. For "western peaches", the individuals perceive the private self as the nucleus of his/her phenomenological world. Based on this axis, the constellation of social relations revolves around it as the fruit and the peel of a peach. By contrast, for onions, there is no core but only a layer after another one. In such a viewpoint, even the ontological ground of "individual" itself is problematic.

From the above models, the key message it intends to make is that under the moral crisis described by $\mathrm{Ci}$, when old doctrines lose their charisma, other scholars try to reformulate the basic constructions while striving to keep the relationally based blueprint. Those attempts are remarkable but tough. When they try to appeal their justifications to the old principles, the practical problem as raised by King and $\mathrm{Ci}$ reappear; but when they abandon them, as what Ames did, the foundation of the methodology is intrinsically intuitivism. If using an analogy from quantum mechanics: the course between the focuses and the fields inevitably are under the "uncertainty principle". In most of the unambiguous situations, this complex intuitive mechanism works. But its validity is questionable in minor cases. The loss of efficiency in those special moments might be the methodological problem in the project of self-formation.

\subsubsection{The Problem of Role Dilemma}

The problem of role-dilemma demonstrates this issue. For most of the cases, the intuitively plausible solutions for them might be based on two preconditions:

1. The unambiguous assumed hierarchical value for different roles.

2. The unambiguous assumed life-sphere where the role performs. 


\subsubsection{The First Type of Role Dilemma.}

The classical example of the first situation perhaps is filial piety, as discussed by Wang [10]. Through its normative influence, it might be intuitively unquestionable that the moral obligations of being a son/daughter are heavier than that of being a citizen. Thus, in an imaginative situation where a person sees both his/her daughter and another girl drowning in a river, despite the role of being a parent and the role of being a citizen is under a conflict, the intuitive answer of solving this dilemma is transparent.

\subsubsection{The Second Type of Role Dilemma.}

The classical example of the second situation perhaps is nepotism [11]. For instance, in the workplace sphere, the expected value of being a citizen, contrary to the previous case, is heavier than being a parent. Consequently, the practice of favoritism by promoting one's son/daughter over another qualified worker is undoubtedly an act of corruption.

However, for situations beyond the qualifications of the above two preconditions. The intuitive approach might fail to solve the dilemma. The most famous example on the cancellation of the first precondition might from Ruth Benedict's classical analysis of Japanese society. In her observation on the story of fortyseven samurais, she argues that for a virtuous Japanese samurai, under the unsolvable pressure from giri and chu, only "in killing themselves with their own hands the ronin paid the supreme debt both to giri and to gimu" [12]. In such a case, the burden of giri is from one's comrades and the prince; the burden of chu is from one's family: their value is almost equal in an intuitive evaluation [13]. When applying Rosemont's logic of onion-self in such an exceptional situation, the conclusion seems to be unethical and counter-intuitive.

In Rosemont's thesis, there is no ontological ground for an individual, thus, "what he/she is" is through the existential relations that he/she faces. Although an onion has no core, it contains inner layers and outer layers. Thus, when there is a clash between two inner-layers, maintaining their intactness is more supreme a mission for the individual than to maintain his/her own life since life without those "inner layers" is groundless and void. Therefore, if self is ontologically role-relational, then, the conflict between two central roles is not only a dilemma but a self-contradiction. Consequently, the action of suicide is not morally wrong but a rather "creative expression" of the self in this holographic field.

One might easily dismiss the above example by arguing it is too extreme. Also, it seems that in modern society under the division of labor, different life-spheres normally have clear-cut boundaries which regulated by detailed disciplines and surveillance [14]. The drastic overlap among different life-spheres in the shogunate is an outdated past. However, as Hartmut Rosa points out that "the multidimensional developments of societal flexibilization, deregulation ... continually augment the number of viable worldly possibilities and options." [15]. The boundaries among different systems, far from moving towards more sophisticated definitions as what in Foucauldian classic modernity, marches to a deregulated flow. As the example of a middle school teacher he uses later:

"When the actor operates in other functional contexts (the university, the citizen's group): the aunt doesn't get invited, the son's bicycle doesn't get repaired, the daughter's birthday party is delayed...social systems ... become 'greedy'... highly temporalized systems tend to become totalitarian." [16].

At this point, it might be clear that the theory of rolerelational self, when faced with the two problems (the practical problem and the methodological problem) is under a dilemma: on the one hand, the old principals are obsolete whose authority on postmodernity no longer holds by itself; on the other hand, the new approaches of being-as-roles lack a basic guideline. The grounding method for the adopters of the new approaches is largely from two resources: empirical examples and intuitions. Because the first category is open to various interpretations, the nature of it is essentially also intuitive.

\section{PSYCHOLOGICAL EXPLANATION}

To deal with this issue, the next task perhaps is to address the dilemma once mentioned concerning the roleself approach. In doing so, it proposes a psychological explanation to the practical problem. Then, to deal with the methodological problem, it tries to adopt a toolbox perspective to combine different models for practical self-formation.

\subsection{Self-Determination Theory and Relatedness}

In a canonical paper on self-determination and motivation, phycologists Ryan and Deci break the old dichotomy between internal commitment and external conformity by proposing a continuum between amotivation and intrinsic motivation. The middle area between the two ends of it is extrinsic motivation by which "whenever an activity is done in order to attain some separable outcome" [17]. For extrinsic motivation, since individuals are not intrinsically interested in the goal that they need to achieve, thus, to build up extrinsically involved behaviors, those behaviors:

"must initially be externally prompted ... by significant others to whom they feel (or would like to feel) connected, whether that be a family, a peer group, or a society. This suggests that the groundwork for facilitating internalization is providing a sense of belongingness and connectedness to the 
persons, group, or culture disseminating a goal, or what in SDT we call a sense of relatedness." [18].

This relatedness has two significant effects. The first one is that it facilitates self-evolvement. The demands of the external goals or principles become a part of the person's own goals or creeds. Therefore, the previous externally regulated behaviors which once are regarded as "controlled or alienated" turn into active "ego involvement", "identification", and "integrated regulation". The other crucial effect accompanies with the first one, which is, through this process of internalization, the behaviors that previously have "external perceived locus of causality" move to an internal one.

The consequences of the two "effects of relatedness" are also two-folded. What Ryan and Deci emphasize is a cognitive one. In another concluding article on their selfdetermination theory, they try to argue that the intrinsically motivated individuals, despite under the seemingly paradoxical needs for relatedness and autonomy, are more willing "to transform socially sanctioned mores or requests into personally endorsed values and self-regulations" [19]. They also unveil more creativity compared with externally motivated individuals.

\subsection{Flow}

The ultimate cognitive result of this intrinsically motivated self is perhaps the experience of flow, as what a psychologist Csikszentmihalyi describes:

"[Flow is] the feeling when things were going well as an almost automatic, effortless, yet highly focused state of consciousness." [20].

Under this condition, individuals experience less apprehensiveness and exert higher creativity.

\subsection{Attributional Theory of Emotion}

Apart from Deci and Ryan's cognitive approach, this internalized self-motivation also might trigger emotional evolvement. In an illuminating experiment conducted by Schachter and Singer [21], they manipulate emotion through attribution. In the experiment, they inject epinephrine (a drug makes people physiologically aroused) to their participants but only tell a part of them the truth. They falsely inform other participants that they are testing a medicine called "suproxin" concerning their eyesight. Then, they create scenarios that tend to make their participants feel euphoric or angry. The general result indicates that when the subjects know they are under the effect of epinephrine, they undergo less emotional change than the participants (the "suproxin" group) do not know the correct reason of their arousal. Therefore, Schachter and Singer conclude that for the informed group, they attribute their physiological arousal to external effects, which is the drug. By contrast, for the non-informed group, they attribute the reason for their physiological arousal as they are influenced by the situation. The consequence of this cognitive attribution is emotive.

\subsection{Apply the Above Theories on Chinese-self}

If reflecting the results from an ethical perspective. It follows that:

For the externalized moral doctrines, without personal involvement, the individuals will deemotionalize themselves by attributing those doctrines as "cold principles".

\subsubsection{An Example in Yang Huо}

Therefore, In Yang Huo (陽貨), when Zai Wo questions the three years' mourning, Confucius asks him "If you were, after a year, to eat good rice, and wear embroidered clothes, would you feel at ease?" [22]. Zai Wo's positive answer disappoints Confucius. When he leaves, Confucius appeals to the emotional affection between parents and children, thus, regarding Zai's emotional reaction as "want of virtue". From the psychological perspective above, the problem of Zai Wo is that: for him, mourning for three years is only a "cold principle" under the guidance of $l i$ (禮). In doing so, he externalizes it from the relatedness to his parents. Cognitively, he cannot actively regulate himself to follow its demand; emotively, he attributes the motif of his behavior as following the external order rather than the physiological arousal triggered by parental love.

\subsubsection{An Example in Water Margin}

By contrast William Sin analyzed the act of $\mathrm{Wu}$ Song's revenge on Pan Jinlian in Water Margins. He argues that this episode is an illustration of ritual killing. In his analysis, Sin points out that:

"Wu Song's interrogation of the culprits, and his execution of Pan are handled with a respectful and solemn attitude, indicating a consideration of li 禮 (rites) in his actions...because of the lack of a clear precedent for such a ritual, $\mathrm{Wu}$ has to creatively perform the rites. " [23].

From this perspective, the origin of the practical problem, when analyzing King's thesis on the relationalself formation, is perhaps due to externalizing/attributing the demand for relational formation as cold principles. In such a way, the guideline of five cardinal relations becomes soulless laws without relatedness. 


\section{SOLUTION}

\subsection{Two Polarized Approaches}

To deal with this problem, the obvious and radical way is de-principalization. As what another school of relational-self tries to do. However, as mentioned before, to attribute everything to intuition or empirical examples will cause the methodological problem.

Moving to the opposite radical dimension, a society might culturally disseminate an internalized ideology to build up the relatedness from a top-down perspective. However, the nature of this action is bio-political and is subject to a post-colonial critique. As what Arif Dirlik points out:

"the hitching of Confucianism to an ideology...ruling out different ways of being 'Chinese' ... its promoters have in some ways completed the process of Orientalizing Confucianism" [24].

The key default of this approach is that it extends an ethical problem to a political agenda. The project of being a self is idiosyncratic but the political demand is a uniformed ideology. In such a way, psychological externalization and Oriental reconstructions [25] are inevitable. However, beyond what Dirlik emphasizes, the dynamics between the subject and the object of this reconstruction is not only the traditional West-East dichotomy, as what Said originally indicated. What is more crucial is the opposition between the representative and the subaltern in a form of epistemic violence as what Gayatri Spivak suggests:

"The small peasant proprietors cannot represent themselves; they must be represented. Their representative must appear simultaneously as their master, as an authority over them" [26].

From the above analysis, it is almost cogent that those extreme ways to settle the practical problem dooms to fail. Only "what in the middle" seems to work. However, under the risk of externalization and de-emotionalization, this middle way cannot be a principle but a method.

\subsection{The Toolbox Perspective}

\subsubsection{The Problem in Li and Xin}

In Wang's [27] article on the genealogical self, before proposing his model, he stages the other three previous ones. Namely: the universal self, the organistic self, and the relational self. In his analysis of the universal and the organistic self-models, he suggests that both models appeal to a transcendental universality or a transcendental oneness. In such a way:

1. The distinctive and lively self is under negation by a universal persona. [28].
2. The centralized self-mind neglects the existence of others.

Wang's analysis is correct. The previous two models hastily appeal to a universal, no matter it is $l i$ (理) or xin (心), their metaphysical essence remains the same. The concrete self, or the self in everyday life, throughout this idealization, recedes to an external abstraction.

But are those approaches useless, or the Chinese-self dooms to circle an unsolvable complex? Perhaps this gloomy rejection is also impetuous. Even though each of them has its flaws, their combinations might not. If the project of self-formation is like crafting an artwork, then, those different models, instead of being an absolute blueprint, might be tools in a kit.

\subsubsection{The Positive and Negative Methods of Fung}

\subsubsection{Fung's Methodology and Problems}

At the end of Fung Yu-Lan's a short history of Chinese philosophy, he acknowledges that "In the history of Chinese philosophy, the positive method [29] was never fully developed; in fact, it was much neglected" [30]. When he wrote this sentence, he perhaps thought mostly about metaphysics. In another essay when he attempts to project the future of Chinese philosophy, although he juxtaposes the significance of the negative method and the ideal life, the comparisons in the latter part of this article are still largely metaphysical. When inquiring about the bedrock of ontology, he writes:

"Reason ... only to find there must be

'something' left, which has no character" [31].

He does not make further implications on ethics. But it logically follows that without the influence of all the external qualifications, there is also "someone" left ethically. And this person, in Fung's philosophy, might be the one in the innocent sphere. The foundation of this person's innocent selfhood does not aim to any abstract metaphysical concept that transcends it; more closely, this innocence portrays everyone's biological origin. What is problematic perhaps is in the later ethical development, Fung moves too fast. When the self crosses the utilitarian sphere to the moral sphere, its theoretical ground implicitly shifts from ethics to politics. In such a way, everything moral becomes external at first glance. And when the self metamorphoses from the moral sphere to the final transcendence, its theoretical ground even leaves politics and heads for the insubstantial metaphysics. In such a way, the true transcendental self is only an ideal.

\subsubsection{Apply Fung's Approach on Li and Xin}

But this drastic change does not undermine the validity of Mr. Fung's methodology. For the neoConfucians like Zhu Xi and Wang Yangming, without the 
criterion of positive inquiry, the negative intuition directly leaps to the highest transcendental ground: their $l i$ and $x i n$ instantaneously resident everywhere but like a ghost, nowhere to find in actuality. When a person acknowledges their emptiness, one question naturally appears: what is the real significance of this transcendental universality? If it is intuitive, purely external, then, the self-request might destinate to an illusionary journey. But the tempting calling of the transcendental self seems always there, like Siren's song, allures but kills.

The answer might be that it indicates the right direction and points to the ultimate finality.

To illustrate this point, considering the most famous paragraph in The Great Learning:

“...Their thoughts being sincere, their hearts were then rectified... Their states being rightly governed, the whole kingdom was made tranquil and happy." [32].

The "transcendental" goal here is the harmony of the kingdom. In the past, it was the pursuit of every Confucian scholar. However, the total tranquil happiness in a state is utopic. This ultimate wish is only a logical end of its sub-requirements rather than what can be achieved in the real world. Nonetheless, without it, the whole procedure that from ethical to political development is incomplete. A similar rationale is also applicable to the Four Spheres. The ever-enlarged selfunderstanding is theoretically possible to cover the whole universe but in practice, it is only a polestar. Nonetheless, without its guidance, the self will estray and get lost.

Therefore, what is of top priority in Mr. Fung's Four Spheres is not its transcendental stages but the way to achieve them, its methodology: the dialectic between the positive and the negative method. What is crucial is that its application should only in an ethical sphere rather than through different theoretical grounds.

Thus, the switch-point from the positive analysis to the negative transcendence should not be at the boundary between ethics and politics nor at the frontline of metaphysics; but should lie in the horizon between the self and others, or among different roles. Under this purely ethical consideration, what is transcendental is not anything universal but the unfathomable gap between "I" and "you"; the manifestation of this gap is through different role relations. If reconsidering Ames' model on focuses and fields, then, a self is both a focus and a field at the same time. When two focuses are in the same field, a "transcendental" relationship begins.

With the beginning of a new relationship, the self leaves the old utilitarian sphere and develops at a new stage. Therefore, what needs here is the positive method again. But the content of this positive method should not be anything external, nor anything transcendental which is inclined to decay into soulless cold principles. On the contrary, it should be something formed but always renewable.

\subsubsection{The Cognitive and Emotive Rules}

The two crafting guidelines of it might from the previously abandoned $l i$ and $x i n$. What is critical here is that this $l i$ should not be the Heavenly Li; nor this xin should be the Heavenly Principle [33] as what originally implied metaphysically. Rather, this $l i$ might be the understanding of a given situation in a role-based relationship; this xin might be the naturally sprouted empathetical commiseration of a certain circumstance in social interactions. Based on that, under a role-relation, the two previous utilitarian selves transcend into a growing "holographic coupling". The two grounding rules in this self-formation project might like that:

1. Cognitively, the two entities under this holistic/holographic coupling should strive to reach situational mutual understanding.

2. Emotively, the two entities under this holistic/holographic coupling strive to channel their arousal to situational mutual resonance.

Like two sides of a coin: the cognitive rule and the emotive rule are inseparable. The mere cognitive understanding without emotional commitment is dangerous. It will evolve into a form of epistemic violence: the accumulated knowledge in those relationships is no other than a tool for control. By contrast, the mere emotive arousal is fatuous and unsustainable. The self, driven by instinctive desires, devolves back to the innocent sphere: in this circumstance, even the utilitarian ego disappears, what remains is only the puerile id. Therefore, the continuation of emotional love has an epistemological ground. This statement is justified both psychologically [34] and philosophically [35].

Therefore, under the conscious guidance of the above rules, a relationship moves to its maturity. The application of the negative method comes consequently. With the internalization of the contextualized discourse between cognitive understanding and emotive commitment, the project of behavioral role-construction reaches to an unconscious flow. Thus, the ultimate condition of a role-relational holographic coupling would be that: without any explicit explanation, there is a mutual understanding; without any implicit hint, there is a reciprocal resonance. At this stage, any analytical progression is paradoxically regressive because at the edge of the boundary, any trial of further exploration is back to the conscious deployment. 


\subsubsection{The Genealogical Model on Chinese- self}

In the mass field of role-reactions, there are many different relationships simultaneously exist. An excellent sketch of it might be Wang's [36] genealogical self. Like what shows up in Chinese characters, the egoistic self is like the "root" of a character. It, on the one hand, marks its origin and is an indispensable part of a character; but on the other hand, it seldomly indicates the completion of it. Under the interplays between the focuses (roots) and the fields (other parts of a character), a pedigree chart of the characters under the same root might be what a real Chinese-self in his/her ethical sphere. More than that, this chart also suggests the limitation of the self-formation process. Because one single root only occurs several times among all the Chinese characters, it is impossible and impractical to over-generalize this self-formation process. The transcendental universal god-like self is too demanding for a single person because a person cannot understand everything around him/her nor build up emotional connections with everyone in a community.

\section{CONCLUSION}

Therefore, to view a Chinese-self under this toolbox approach, it seems that the component of understanding in the universal-self and the component of sentimentality in the organistic-self become its two basic rules; the course of the role relational-self becomes its ontological base; the genealogical-self becomes its objectives and its manifestation. Although this approach has many limits even practically, it, at least, sheds a light on the intricate project of self-formation by addressing the practical problem and the methodological problem. Using Bernard Williams' [37] terminology, it prevents the practical problem mainly by not appealing to any soulless abstract thin principles but to the action-guiding thick concepts. It deals with the methodological problem mainly by readopting Fung's positive and negative methods into a pure role-based ethical sphere. Under this construction, the self regains an ontological base. The previous "onion model" raised by Rosemont might be replaced by a "chemical compound" model, in which a self and the other are different molecules. The different degrees of understanding and resonance is perhaps the energy of the chemical bonds in each compound. When under role conflicts, if all the particles are organized through mutual understanding and resonance, through the "the principle of minimum energy", a new chemical compound will naturally come into being without the negation of any individual particle.

\section{REFERENCES}

[1] [27] [36] Wang, Qingjie. "Genealogical Self and a Confucian Way of Self-Making." International philosophical quarterly 42, no. 1 (2002): 93-112. https://doi.org/https://doi.org/10.5840/ipq20024217 4.

[2] Ambrose King, "The Individual and Group in Confucianism: A Relational Perspective," in Individualism and Holism: Studies in Confucian and Taoist Values (Ann Arbor, Michigan: University of Michigan Press, 1985), pp. 57-70, 57,63-4.

[3] Confucius. “The Analects: Yan Yuan.” Translated by James Legge, 2006. https://ctext.org/analects/yanyuan.

[4] Ci Jiwei, The Moral Crisis in Post-Mao China: Prolegomenon to a Philosophical Analysis, (Zürich, Swiss: Diogenes, 2009), 15.

[5] Tang, Junyi. The Complete Works of Tang Junyi. Taipei, Taiwan: Xuesheng Shuju, 1991.

[6] [8] David L. Hall and Roger T. Ames, Thinking Through Confucius (New York City, New York: State University of New York Press, 1987), 237, 262.

[7] Roger T. Ames, Confucian Role Ethics: a Vocabulary (Hong Kong, Hong Kong: The Chinese University Press, 2012), 131.

[9] Henry Rosemont, Against Individualism: a Confucian Rethinking of the Foundations of Morality, Politics, Family, and Religion. (Lanham, Maryland: Lexington Books, 2016), 94.

[10] Wang, Qingjie. "The Confucian Filial Obligation and Care for Aged Parents." Essay. In Confucian Bioethics, 235-56. Dordrecht, Netherlands: Springer, 1999.

[11] See Wang's response to the practical problem in Golden Rule, Wang, Qingjie James. "The Golden Rule and Interpersonal Care: From a Confucian Perspective." Philosophy East and West 49, no. 4 (1999): 415. https://doi.org/10.2307/1399946.

[12] Ruth Benedict, The Chrysanthemum and the Sword: Patterns of Japanese Culture (Boston, Massachusetts: Houghton Mifflin Company, 1989), 205.

[13] The similar situation occurs when Confucius places a parallel between the government and the family through his famous saying "There is government, when the prince is prince, and the minister is minister; when the father is father, and the son is son." See Confucius. "The Analects: Yang Huo." Translated by James Legge, 2006. https:/ctext.org/analects/yang-huo

[14] For example, see Michel Foucault, Discipline and Punish: The Birth of the Prison. (New York City, New York: Vintage, 2009), 297. 
[15] [16] Hartmut Rosa, Social Acceleration: a New Theory of Modernity, trans. Jonathan Trejo-Mathys (New York, New York: Columbia University Press, 2013), 184, 188-191.

[17] [18] Richard M. Ryan and Edward L. Deci, "Intrinsic and Extrinsic Motivations: Classic Definitions and New Directions," Contemporary Educational Psychology 25, no. 1 (2000): pp. 54-67, https://doi.org/10.1006/ceps.1999.1020, 60, 64.

[19] Edward L. Deci and Richard M. Ryan, "The 'What' and 'Why' of Goal Pursuits: Human Needs and the Self-Determination of Behavior," Psychological Inquiry 11, no. 4 (2000): pp. 227-268, https://doi.org/10.1207/s15327965pli1104 01, 235236.

[20] Mihaly Csikszentmihalyi, Creativity: Flow and the Psychology of Discovery and Invention (New York, New York: HarperCollins Publishers, 1996), 110.

[21] Schachter, Stanley, and Jerome Singer. "Cognitive, Social, and Physiological Determinants of Emotional State." Psychological Review 69, no. 5 (1962): 379-99. https://doi.org/10.1037/h0046234.

[22] Confucius. "The Analects: Yang Huo." Translated by James Legge, 2006. https://ctext.org/analects/yang-huo.

[23] William Sin, "Wu Song's Killing of His Sister-inLaw: An Ethical Analysis," Dao 17, no. 2 (June 2018): pp. 231-246, https://doi.org/10.1007/s11712018-9603-0, 232-233.

[24] Dirlik, Arif. Culture \& History of Postrevolutionary China: The Perspective of Global Modernity (Hong Kong, Hong Kong, The Chinese University of Hong Kong Press, 2011), 153-154.

[25] See Said, Edward W. Orientalism: Edward W. Said. New York, New York: Vintage Books, 2003.

[26] Gayatri Chakravorty Spivak, "Can the Subaltern Speak?" in Colonial Discourse and Post-Colonial Theory: A Reader, ed. Patrick Williams and Laura Chrisman (New York, New York: Columbia University Press, 1994), pp. 66-111, 71.

[28] This is basically from his analysis of Song neoConfucianism, where the ultimate universal is governed by $l i$ (理) under which, other idiosyncratic manifestations like $q i$ (气) and xing (性) are just its representations. See Zhu Zi Yu Lei chapter 1 section 4; chapter 1 section 10; chapter 5 section 8 etc. Zhu, Xi. "Zhu Zi Yu Lei." Chinese Text Project, 2006. https://ctext.org/zhuzi-yulei.
[29] The positive method is about logical analysis; the negative method is to remain silent when touches the logical boundary.

[30] Yu-lan Fung, A Short History of Chinese Philosophy, ed. Derk Bodde (New York City, New York: Macmillan, 1966), 342.

[31] Yu-lan Fung, "Chinese Philosophy and a Future World Philosophy," The Philosophical Review 57, no. 6 (1948): pp. 539-549, 541.

[32] Dai, Sheng. "Da Xue." Translated by James Legge, 2006. https://ctext.org/liji/da-xue.

[33] Wang Yangming is correct in a way by recognizing the biologically encoded xin for almost all the people. However, his drastic refusal of selfish desire by appealing to the heavenly principle of xin is a hasty metaphysical assertion. Both Wang and Zhu, in this way, negate the self. Zhu is through a cognitive approach; Wang is more like an emotive approach. As he said "The heart-mind is principle. When this heart-mind is kept completely free of the slightest impediment from selfish desire, then it is Heavenly principle." Xiangshan Lu et al., Readings from the Lu-Wang School of Neo-Confucianism (Indianapolis, Indiana: Hackett, 2009), 138.

[34] Sternberg, Robert J. "A Triangular Theory of Love." Psychological Review 93, no. 2 (1986): 119-35. https://doi.org/10.1037/0033-295x.93.2.119.

[35] See the last chapter on love and understanding in Nishida, Kitarō. An Inquiry into the Good. Translated by Masao Abe. New Haven, Connecticut: Yale University Press, 1992.

[37] Williams, Bernard. Ethics and the Limits of Philosophy. London, England: Routledge, 2011. 